\title{
Normativa, gestión de riegos y experiencia sobre depósitos de relaves en Chile
}

\author{
MariaTeresa Zandarín Iragorre \\ JUniversidad Técnico Federico Santa María, Campus San Joaquín, Santiago de Chile. \\ maria.teresa.zandarin@gmail.com
}

\begin{abstract}
RESUMEN
Chile es el primer productor de cobre mundial, la producción de cobre fino en el 2018 alcanzó aproximadamente las 5,5 Mton se estima que en el año 2025 se produzca 7,2 Mton de cobre anual. Esta producción, sumada a leyes de cobre inferiores al 1\%, provoca que el $99 \%$ del material que se procesa se convierta en pasivo ambiental, entre depósitos de relaves, pilas de lixiviación y depósitos de ripios. Los relaves están clasificados como pasivos ambientales de alto riesgo, y además se ubican en un país de alta sismicidad. Es por esto que en Chile existe un marco regulatorio que ha permitido mejorar los estándares en el diseño, construcción y operación de los depósitos de relaves fundado en la experiencia sobre el comportamiento de estas estructuras. Además, en la actualidad se está implementando un sistema de gestión de riesgos que permita tempranamente identificar fallas para tomar decisiones y acciones que permitan asegurar la estabilidad física y química de estos depósitos. En este artículo se describirá la experiencia sobre el comportamiento de depósitos de relaves sometidos a eventos sísmicos de alta magnitud, se comentará brevemente el marco regulatorio y la gestión de riesgos vigente.
\end{abstract}

Palabras clave: depósito, geotécnia, normativa, relaves, sísmica.

\section{Regulations, risk management and experience about tailings dams in Chile.}

\begin{abstract}
Chile is the biggest copper producer in the world, the production of fine copper in 2018 was approximately 5.5 Mton and it is estimated that in 2025, 7.2 Mton of copper will be produced. This production, added to copper grades of less than 1\%, causes $99 \%$ of the material that is processed to become mine waste such as tailings storage, heap leach pads and rubble deposits. Tailings are classified as high risk environmental waste, and these tailings are also located in a country of high seismicity. Due to this, Chile has a regulatory framework that allowed the improvement of the standards in the design, construction and operations of tailings deposits based on the know-how about the behaviour of these structures. Currently, a risk management system is being implemented to identify the risks and take decisions and actions to ensure the physical and chemical stability of these different types of storage. This article describes the Chilean experience about the behaviour of tailings storage under high magnitude seismic events. A summary about the regulatory framework and the recently implemented risk management is also presented.
\end{abstract}

Keywords: geotechnical; regulation; seismic; storage; tailings 


\section{Introducción}

El Gobierno de Chile a través del Servicio Nacional de Geología y Minería (Sernageomin) tiene catastrado 740 depósitos de relaves (marzo 2018), de los cuales 469 se encuentran inactivos, 170 abandonados y 101 están en operación. La tecnología de depositación más utilizada es la convencional (relaves con contenidos de sólido entre $38 \%$ y $50 \%$ ) con el uso de muros de arenas (muros construidos con la fracción gruesa del relave). Estos depósitos reciben el nombre de Tranque de Relaves y representan el $80 \%$ de los depósitos que existen en Chile. Le siguen en importancia (16\%) los embalses de relaves, en los que el muro de contención está construido por materiales de empréstitos. En el resto de los depósitos se utiliza la tecnología de relaves en pasta, espesados y filtrados. El volumen de relaves que acumulan actualmente los depósitos en operación es igual a $6.4 \mathrm{Mm}^{3}$. Y el volumen total aprobado para estos depósitos es igual a $14,3 \mathrm{Mm}^{3}$ (6.600 millones de toneladas). (Sernageomin, 2018). Esta realidad sumada a la alta sismicidad de Chile, a la últimas fallas ocurridas en depósitos de relaves y al involucramiento activo de las comunidades en los proyectos mineros han ocasionado el origen de normativas cada vez más exigentes y en sistemas de gestión de riesgos que aseguren el diseño, construcción y operación segura de depósitos de relaves. Asimismo, se han generado nuevas líneas de investigación para minimizar el impacto de los relaves al medioambiente.

En este artículo se describe brevemente la experiencia chilena sobre diseño, operación y comportamiento de los depósitos de relaves. También se presenta la evolución de las normativas chilenas respecto a relaves debido a la ocurrencia de fallas de depósitos que han provocado fatalidades.

\section{Fallas ocurridas en tranques de relaves}

En Chile se han registrado 38 casos de fallas de tranques de relaves (muros de arena) debido a licuefacción sísmica, inestabilidad de taludes símica y overtopping. El $50 \%$ de las fallas se deben a licuefacción, el
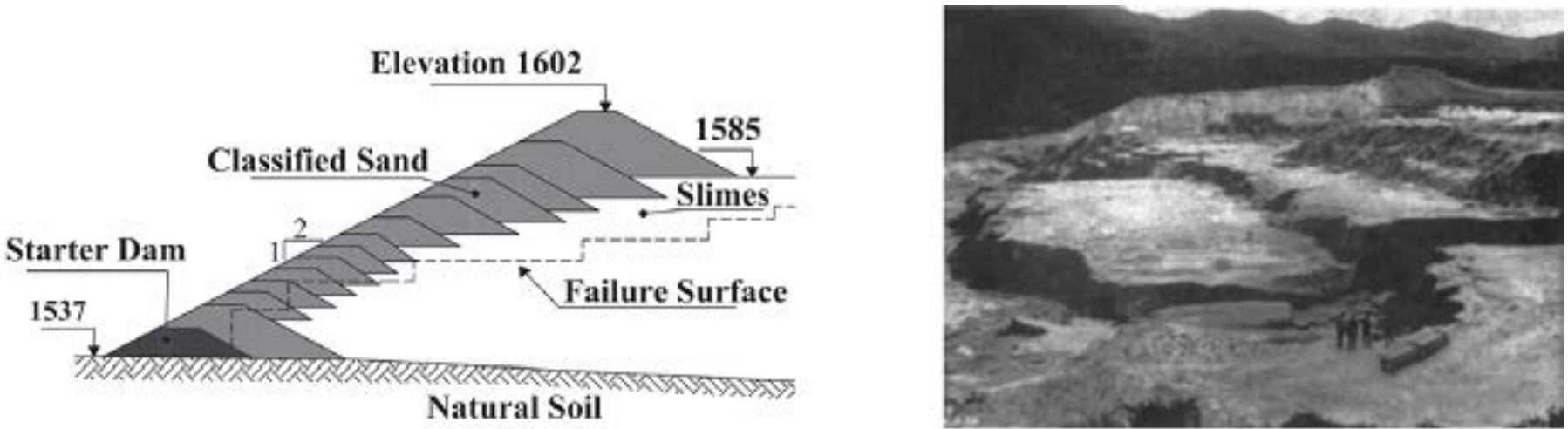

Figura 1. Sección delTranque Barahona antes y después del sismo. (Troncoso et al., 1993).

Fiqure 1. Cross section of Barahona tailinas dam before and after the earthauake. (Troncoso et al., 1993).

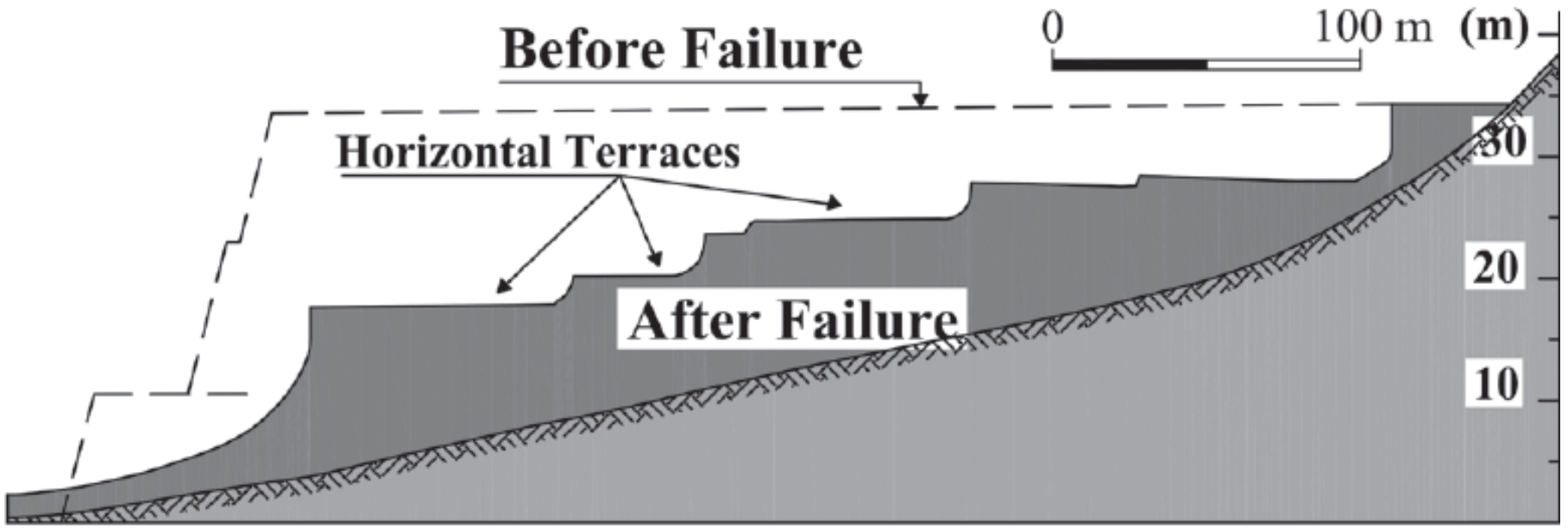

Figura 2. Sección del Tranque el cobre antes y después del sismo (Troncoso et al., 1993).

Figure 2. Cross section of El Cobre tailings dam before and after the earthquake. (Troncoso et al., 1993). 
$32 \%$ a inestabilidad de taludes y el $18 \%$ a overtopping seguido de una falla por flujo. Las fallas producidas por los sismos corresponden al $68 \%$ de los casos registrados mundialmente. Las características comunes de los casos registrados en Chile son en muros de arenas ciclonadas de altura mediana entre 15 a $65 \mathrm{~m}$, construidos por el método de aguas arriba, con deficiencias en su compactación y taludes empinados con relación 1,2:1 y 1,7:1(Horizontal: Vertical) (Villavicencio et al. 2013).

Una de las fallas de flujo más antiguas registrada de un depósito de relaves corresponde a la falla del Muro del Tranque Barahona, debido al sismo de magnitud Richter 8.2 que ocurrió el 1 de diciembre de 1928. El muro del depósito, al momento del evento sísmico, tenía $65 \mathrm{~m}$ de altura y una longitud de $1.885 \mathrm{~m}$ colapsó 3 minutos después de ocurrido el sismo. La falla de este muro provocó el vertido de $2,8 \mathrm{Mm}^{3}$ de material que fluyó hacia el valle del río Coya. Como consecuencia de esta falla 54 personas murieron. La sección inicial final del depósito se muestra en la Figura 1. La falla se produjo debido a la licuación de las lamas (fracción fina de los relaves) sobre los que se construía el muro (Troncoso et al., 1993).

Otra falla catastrófica se produjo durante el terremoto de del 28 de marzo de 1965 (magnitud Ms = 7.4), en el tranque de relaves denominado El Cobre. En este evento 2 Mton de material fluyeron $12 \mathrm{~km}$ en pocos segundos, destruyendo el pueblo El Cobre y matando a más de 200 personas. En el momento de la falla el muro del depósito tenía 33 metros de altura y su talud aguas abajo tenía una pendiente entre $35^{\circ}$ y $40^{\circ}$ respecto de la horizontal. Las secciones antes y después de la falla se muestran en la Figura 2 (Dobry et al., 1967).

Luego, durante el terremoto del 3 de marzo de 1985, cuya magnitud fue de 7.8 , dos tranques de relaves fa-

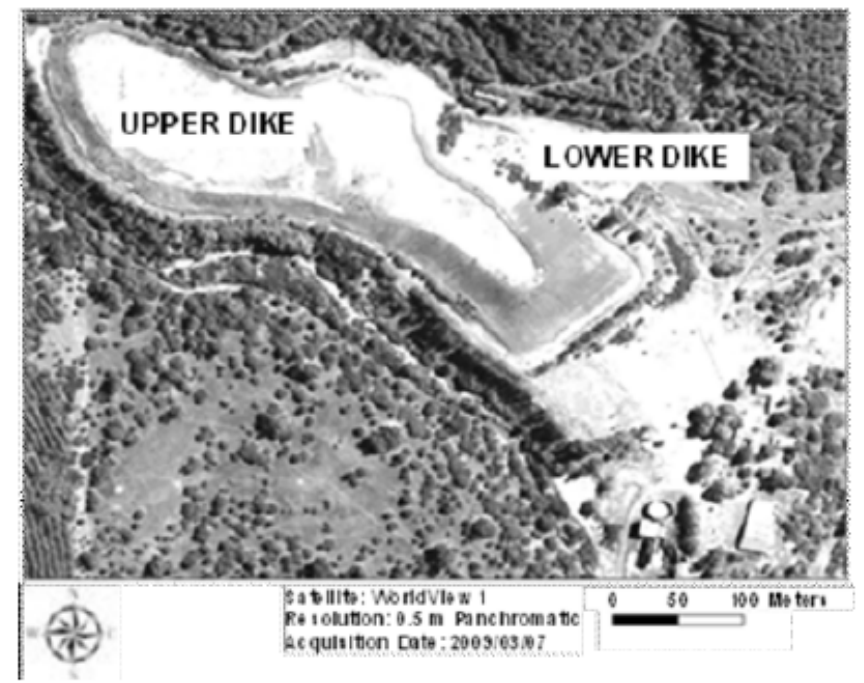

Figura 4. Falla delTranque Las Palmas (Bray et al., 2011).

Figure 4. Flow failure of Las Palmas tailings dam (Bray et al., 2011).

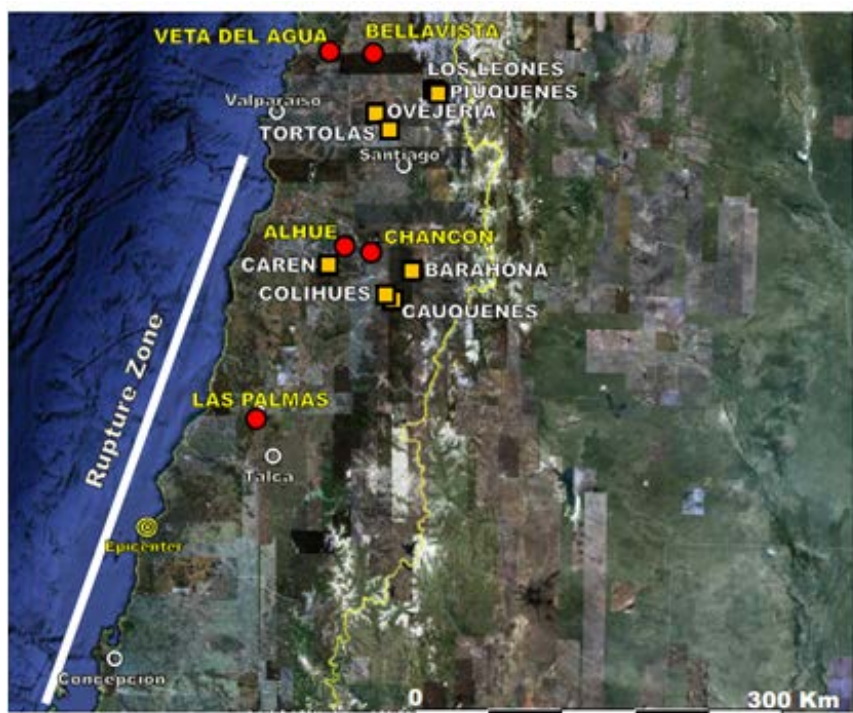

Figura 3. Zona de Ruptura sismo Maule y ubicación de Tranques de Relaves. Con círculos se indican los tranques que fallaron y con cuadrados los que no presentaron fallas (Verdugo et al., 2010).

Figure 3. Fault zone and tailings dams. The tailings dams which were damaged are indicated with circles and those that were not damaged are indicated with squares (Verdugo et al., 2010).

Ilaron por licuación. Una de las fallas se presentó en el Muro Cerro Negro, tenía $30 \mathrm{~m}$ de altura y se vertieron 130 mil toneladas de relaves, que fluyeron $8 \mathrm{~km}$ aproximadamente. La otra falla ocurrió en el muro Veta de Agua $\mathrm{N}^{\circ} 1$, el que tenía una altura de $15 \mathrm{~m}$. La falla ocurrió en la parte central del muro, minutos después que terminó el sismo. Los relaves almacenados fluyeron $5 \mathrm{~km}$ por el lago El Sauce (Castro et al., 1989).

El registro más reciente del comportamiento de los tranques de relaves en Chile se obtuvo luego de la ocurrencia del sismo del 27 de febrero de 2010 en la

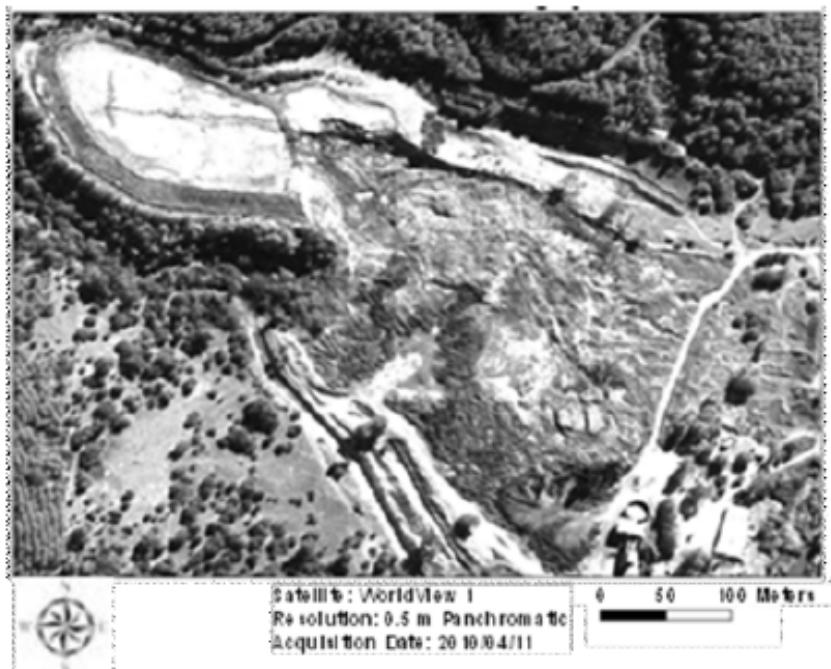




\begin{tabular}{|c|c|c|c|c|c|}
\hline $\begin{array}{l}\text { Nombre del } \\
\text { Depósito }\end{array}$ & $\begin{array}{l}\text { Tipo de } \\
\text { Depósito }\end{array}$ & Método Constructivo & $\begin{array}{l}\text { Altura máxima } \\
(2010) \\
(\mathrm{m}) \\
\end{array}$ & $\begin{array}{c}\text { Taludes } \\
(\mathrm{H}: \mathrm{V})\end{array}$ & Estado \\
\hline Carén & Embalse & $\begin{array}{l}\text { Aguas Abajo } \\
\text { Muro Zonado con núcleo } \\
\text { impermeable y enrocado talud } \\
\text { aguas abajo }\end{array}$ & & $1.8: 1$ & Operativo \\
\hline Colihues & $\begin{array}{l}\text { Embalse } \\
\text { formado por } 3 \\
\text { Muros }(A, B, C)\end{array}$ & $\begin{array}{l}\text { Aguas Abajo. Muros del tipo } \\
\text { zonado con materiales } \\
\text { empréstito. }\end{array}$ & $\begin{array}{l}\text { Muro } A=83 \mathrm{~m} \\
\text { Muro } B=42 \mathrm{~m}\end{array}$ & $2.2: 1$ & $\begin{array}{c}\text { No } \\
\text { Operativo }\end{array}$ \\
\hline Cauquenes & $\begin{array}{l}\text { Tranque } \\
\text { formado por } 5 \\
\text { Muros }(0,1,2,3 \\
\text { v } 4)\end{array}$ & $\begin{array}{l}\text { Aguas Abajo y Aguas Arriba, } \\
\text { Muro de Arenas reforzado con } \\
\text { material fluvial }\end{array}$ & $\begin{array}{l}\text { Muro } 1=51 \mathrm{~m} . \\
\text { Muro 2 }=35 \mathrm{~m} .\end{array}$ & $3.5: 1$ & $\begin{array}{c}\text { No } \\
\text { Operativo }\end{array}$ \\
\hline $\begin{array}{l}\text { Barahona } \\
\text { Muro } 1 \text { y } 2\end{array}$ & Tranque & $\begin{array}{l}\text { Aguas Abajo y Peraltes Aguas } \\
\text { Arriba/ Muro } 2 \text { con refuerzo con } \\
\text { arenas gruesas compactadas } \\
\text { cubierta con escorias. } \\
\text { Muro con refuerzo de enrocado }\end{array}$ & $\sim 65 \mathrm{~m}$ & $2.5: 1$ & $\begin{array}{c}\text { No } \\
\text { Operativo }\end{array}$ \\
\hline Los Leones & Embalse & Núcleo de tierra & $128 \mathrm{~m}$ & & $\begin{array}{c}\text { No } \\
\text { Operativo }\end{array}$ \\
\hline Piuquenes & Tranque & & $55 \mathrm{~m}$ & & $\begin{array}{c}\text { No } \\
\text { Operativo }\end{array}$ \\
\hline Ovejeria & Tranque & Aguas Abajo. Muros de Arena & $58 \mathrm{~m}$ & & Operativo \\
\hline La Tórtolas & Tranque & Aguas Abajo. Muro de Arenas & & & Operativo \\
\hline
\end{tabular}

Tabla 1. Características de los depósitos de relaves que no presentaron falla durante el sismo de Maule.

Table 1. Main characteristics of the tailings deposits that were not damaged during the Maule earthquake.

Región del Maule. El terremoto de Maule tuvo una $M w=8.8$ y afectó a la zona central y sur de Chile. Para este sismo se registró una aceleración máxima en roca de $0,32 \mathrm{~g}$ y su duración fue de aproximadamente 2 minutos. Este sismo generó entre la placa de Nazca y la placa Sudamericana una zona de ruptura que se extendió entre las ciudades de Concepción y San Antonio, es decir, $500 \mathrm{~km}$ de longitud aproximadamente. En la Figura 3 se muestra la zona de ruptura respecto a la ubicación de los tranques de relaves que se inspeccionaron luego del sismo (Verdugo et al., 2012). En esta ocasión se inspeccionaron: Embalse Carén (en operación, Muro de empréstito), Tranque Colihues (no operativo), Tranque Cauquenes (no operativo), Tranque Barahona (no operativo), Los Leones (no operativo), Piuquenes (no operativo), Tranque Ovejería (operativo) y Tranque Las Tórtolas (operativo). En la Tabla 1 se presenta las principales características de los muros anteriormente indicados. Ninguno de los depósitos mencionados presentó problemas de estabilidad. Sin embargo, cinco muros construidos con el método de aguas arriba presentaron distintos niveles de daño (Chacón, Bellavista $N^{\circ} 1$, Veta del Agua $N^{\circ} 5$, Alhué, y Las Palmas). Las Palmas fue el tranque que presentó la falla más catastrófica. El depósito estaba formado por dos muros, uno ubicado aguas arriba del otro. Ambos muros se construyeron por etapas utilizando el método de aguas arriba. Durante el terremoto los relaves contenidos por el muro de aguas abajo licuaron produciéndose una brecha en el lado este del depósito que permitió el vertido de 100,000 $\mathrm{m}^{3}$ de material que fluyeron $0,5 \mathrm{~km}, \mathrm{y}$ matando a 5 personas (Figura 4).

\section{Normativa chilena sobre depósitos de relaves}

En Chile existe normativa que regula específicamente las distintas fases de diseño, construcción, operación y cierre de los depósitos de relaves. La primera normativa respecto a relaves en Chile es el Decreto Supremo (DS) N 86 "Reglamento de Construcción y Operación de tranques de Relaves", el que se promulgó el año 1970. Luego de la ocurrencia de la falla de El Cobre mencionada en el capítulo anterior. En este decreto se prohíbe el uso del método de construcción hacia aguas arriba de los crecimientos de los depósitos. Este decreto fue reemplazado en el año 2007 por el DS Nº 248 (Tabla 2). El decreto indica que los mé- 
todos y procedimientos de cada proyecto de depósito de relave deben garantizar la estabilidad física y química del depósito y su entorno, con el fin de proteger a las personas, bienes y medio ambiente. En este último decreto se establecen límites por ejemplo para: el contenido de finos ( $20 \%$ de partículas bajo malla 200) de las arenas ciclonadas a utilizar en la construcción de los muros, revancha mínima igual a $1 \mathrm{~m}$ entre el nivel de coronamiento y el nivel de la laguna operacional, factor de seguridad (FS) pseudo-estático mayor a 1,2. Y también indica que el prisma resistente del depósito (muro de contención) debe estar instrumentado para controlar su comportamiento físico (asentamientos, deslizamientos, niveles freáticos entre otros).

Además, los tranques de relaves que superen los $5,000 \mathrm{~m}^{3}$ deben ser aprobados por la Dirección General de Aguas (DGA). El DS N 50 del año 2015 (Tabla 2), indica los requisitos solicitados por la DGA para que un depósito de relaves convencional sea aprobado. En este decreto se clasifican los depósitos en categorías dependiendo de su volumen y altura de muro (Categoría A: Pequeños, de altura de muro máxima mayor a $5 \mathrm{~m}$ e inferior a $15 \mathrm{~m}$, o bien de capacidad superior a $50.000 \mathrm{~m}^{3}$ e inferior a $1.500 .000 \mathrm{~m}^{3}$. Categoría B: Medianos, de altura de muro máxima mayor o igual a $15 \mathrm{~m}$ e inferior a $30 \mathrm{~m}$, o bien de capacidad igual o superior a $1.500 .000 \mathrm{~m}^{3} \mathrm{e}$ inferior a 60.000 .000 $\mathrm{m}^{3}$. Y Categoría C: Grandes, de altura máxima de muro igual o superior a $30 \mathrm{~m}$, o bien de capacidad igual o superior a $60.000 .000 \mathrm{~m}^{3}$.). Establece los estudios previos a realizar. Y acorde a la categoría del depósito también establece un marco para calcular las cecidas máximas probables y la estabilidad física estática y pseudo-estática considerando Sismo Máximo Creíble y Sismo de Diseño, indicando factores de seguridad mínimos a cumplir (FS Estático mayor a 1,4; FS pseudo-estático mayor igual a 1,2; FS post símico mayor

\begin{tabular}{|c|c|c|c|c|}
\hline Norma & $\begin{array}{l}\text { Tipo de } \\
\text { Norma }\end{array}$ & Entidad & Nombre de la Norma & $\begin{array}{l}\text { Año de } \\
\text { promulgación }\end{array}$ \\
\hline $\mathrm{DS} \mathrm{N}^{\circ} 248$ & Reglamento & $\begin{array}{l}\text { Ministerio } \\
\text { de Minería }\end{array}$ & $\begin{array}{l}\text { Reglamento para la aprobación de proyectos de diseño, } \\
\text { construcción, operación y cierre de los depósitos de } \\
\text { relave. }\end{array}$ & 2007 \\
\hline DS $N^{\circ} 50$ & Reglamento & $\begin{array}{l}\text { Ministerio } \\
\text { de Obras } \\
\text { Públicas }\end{array}$ & $\begin{array}{l}\text { Reglamento que se refiere al artículo } 295 \text { inciso } 2^{\circ} \text {, del } \\
\text { código de aguas, estableciendo las condiciones } \\
\text { técnicas que deberán cumplirse en el proyecto, } \\
\text { construcción y operación de las obras hidráulicas } \\
\text { identificadas en el artículo } 294 \text {. (Exceptúa relaves } \\
\text { pasta, filtrados y espesador con Cp mayor al } 65 \% \text { ) }\end{array}$ & 2015 \\
\hline Ley 19.300 & Ley Marco & $\begin{array}{l}\text { Ministerio } \\
\text { de Medio } \\
\text { Ambiente }\end{array}$ & Ley sobre Bases Generales del Medioambiente. & 1994 \\
\hline $\operatorname{DS~N}^{\circ} 148$ & Reglamento & $\begin{array}{l}\text { Ministerio } \\
\text { de Salud }\end{array}$ & $\begin{array}{l}\text { Reglamento sanitario sobre manejo de residuos } \\
\text { peligrosos. }\end{array}$ & 2003 \\
\hline $\mathrm{DS} \mathrm{N}^{\circ} 40$ & Reglamento & $\begin{array}{l}\text { Ministerio } \\
\text { de Medio } \\
\text { Ambiente }\end{array}$ & $\begin{array}{l}\text { Reglamento del Sistema de Evaluación de Impacto } \\
\text { ambiental. }\end{array}$ & 2013 \\
\hline Ley 20.551 & Ley & $\begin{array}{l}\text { Ministerio } \\
\text { de Minería }\end{array}$ & Regula el cierre de faenas e instalaciones mineras. & 2011 \\
\hline
\end{tabular}

Tabla 2. Normativa chilena que aplica para los depósitos de relaves.

Table 2. Chilean regulations for tailings storage. 
1,0). Requiriendo para los depósitos de Categoría C análisis dinámico considerando un sismo de magnitud $\mathrm{Ms}=8,5$.

Y finalmente, para terminar con la operación de los tranques de relaves SERNAGEOMIN debe aprobar un Plan de Cierre el que está regulado a su vez por la Ley $N^{\circ} 20.551$.

Además, todos los proyectos mineros deben superar una Evaluación de Impacto Ambiental o Declaración de Impacto Ambiental dependiendo de las dimensiones del proyecto o del impacto que este ocasione. En esta evaluación el proyecto es examinado y luego de pasar por varias etapas intermedias en las que distintos órganos sectoriales revisan el proyecto, este puede ser aprobado, objetado o rechazado. Es parte de esta evaluación realizar una Línea Base de flora, fauna, agua, suelo y aire en cada estación del año. En este caso se aplica la Ley 19.300 sobre Bases Generales del Medioambiente. En la Tabla 2 se resumen las normativas que aplican en Chile para los depósitos de relaves.

\section{Buenas prácticas aplicadas en tranques de relaves}

Como se mencionó con anterioridad, durante el sismo de Maule (2010), los tranques que se encontraban operativos y los que se diseñaron con crecimiento hacia aguas abajo no presentaron fallas. Además, todos los tranques operativos se diseñaron en el marco normativo del DS $\mathrm{N}^{\circ} 86$. A continuación se describen las principales características de los tranques Las Tórtolas y Ovejería.

El Tranque Las Tórtolas se ubica a $45 \mathrm{~km}$ al norte de la ciudad de Santiago. Los estudios de ingeniería de este tranquen se iniciaron en el año 1984. El Muro Principal del tranque se diseñó para una altura máxima de $150 \mathrm{~m}$. El diseño original consideraba un porcentaje de finos menor a la \#200 máximo del 10\%. EI diseño consideró la implementación de una red de instrumentos geotécnicos que incluyó piezómetros y acelerómetros. Este tranque comenzó a operar en el año 1992 y se decidió años más tarde incrementar el contenido de fino de las arenas a $15 \%$ e incrementar la altura del muro a $170 \mathrm{~m}$, debido a su buen desempeño en cuanto a estabilidad física e hídrica, comprobada con la red de instrumentos. Este tranque también tiene otros dos muros de arena con crecimiento hacia aguas abajo. La cubeta tendrá una capacidad para almacenar 2.000 millones de toneladas de lamas. La construcción del Muro Principal comenzó con la construcción de un muro de partida de material de empréstito compactado hasta una altura de $17 \mathrm{~m}$. Para construir la fundación de este muro se reemplazaron 5 metros de suelo aluvial y se realizó compactación dinámica para evitar que en la fundación quedaran suelos aluviales de baja compacidad. El resto de los suelos de fundación son gravas arenas densas. En la fundación del muro también se construyó un sistema basal de drenaje robusto. Y el crecimiento del muro se construyó con arenas ciclonadas compactadas al $95 \%$ de la densidad máxima seca del Proctor Standard. El talud aguas abajo del muro se construyó formando una pendiente igual a 1:4 (Vertical: Horizontal). En la Figura 5 se muestra la sección del Muro Principal de Las Tórtolas.

EI Tranque Ovejería está ubicado en Huechún a 50 $\mathrm{km}$ al norte de Santiago. Este tranque comenzó su operación en el año 1992. Y su muro principal es un muro de arena cuyo muro de partida tiene $16 \mathrm{~m}$ altura y fue construido con distintos tipos de suelos de empréstito. El talud aguas abajo del muro de partid tenía pendientes de 1,75:1 y 4:1 (Horizontal: Vertical) para facilitar la construcción del muro de arenas. Sobre este muro de partida se depositó hidráulicamente arenas ciclonadas con un contenido máximo de partículas menores a \#200 igual al 15\%. El muro principal tiene 56 metros de altura, un ancho de coronamiento de $20 \mathrm{~m}$ y un talud igual a 4:1 (Horizontal: Vertical). Este muro tiene un sistema de drenaje que permite que el agua contenido en las arenas percole hasta alcanzar la humedad óptima para su compactación. El grado de compactación del muro es superior al $95 \%$ de la densidad máxima seca del Proctor Standard. En la Figura 6 se muestra una sección del Muro Principal delTranque Ovejería.

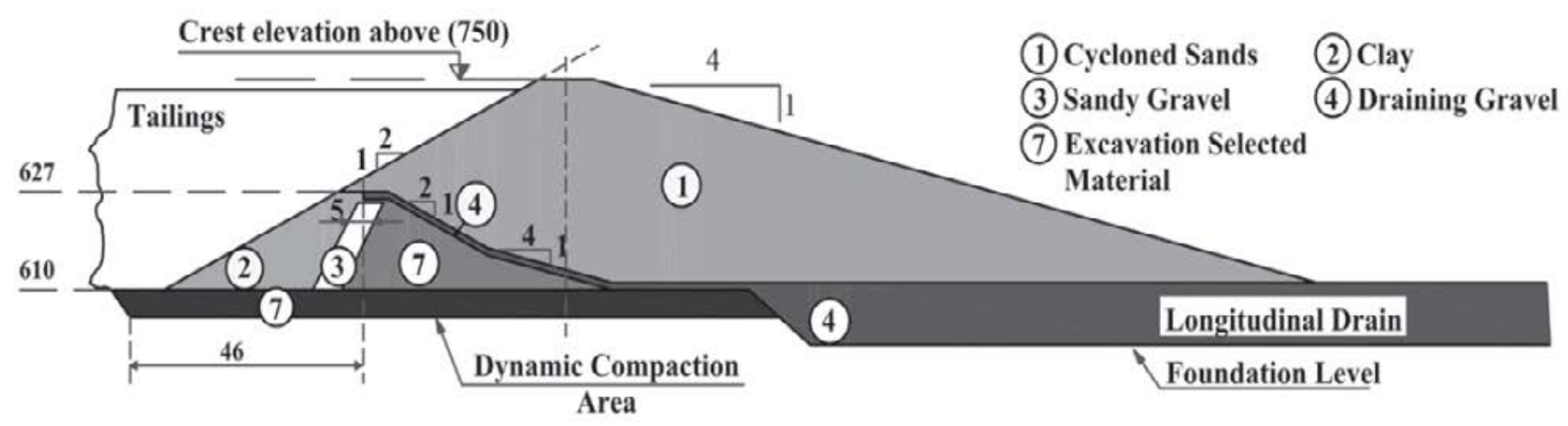

Figura 5. Sección Transversal Muro Principal Tranque Las Tórtolas (Valenzuela, 1996).

Figure 5. Cross-section of the main dams of the Las Tórtolas tailings deposit (Valenzuela, 1996). 


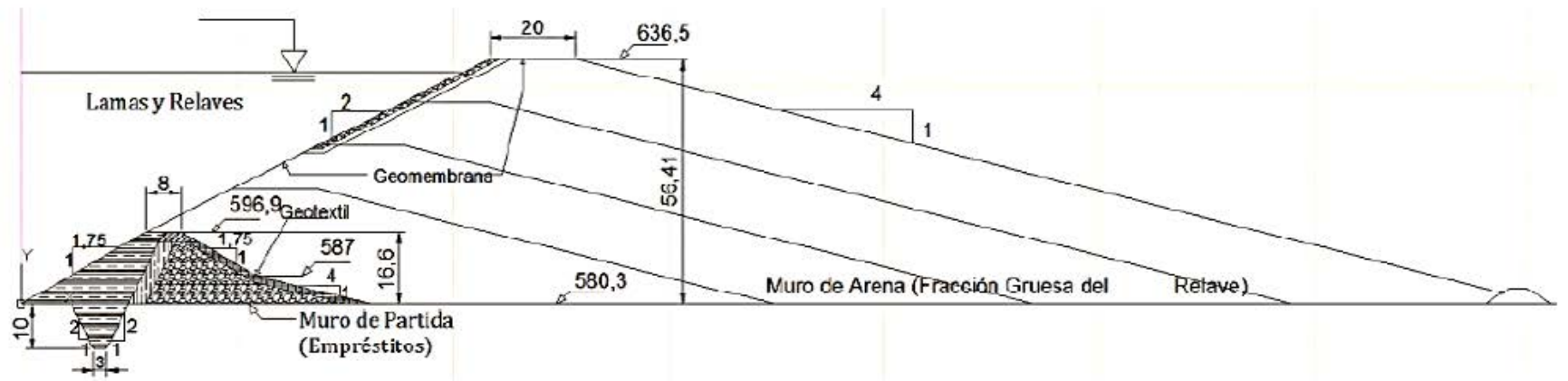

Figura 6. Sección Transversal Muro PrincipalTranque Ovejería (Barrios, 2017).

Figure 6. Cross-section of main dams of the Ovejería tailings deposit (Barrios, 2017).

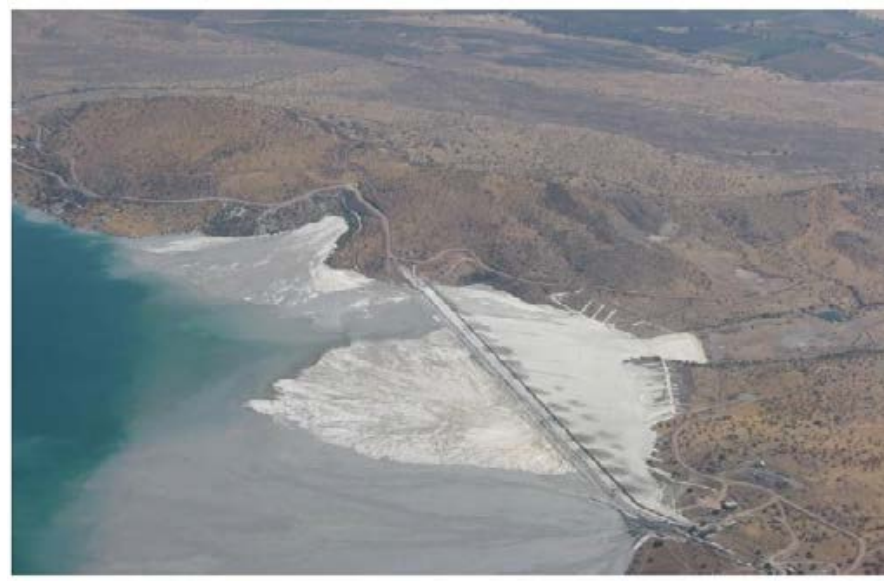

Figura 7. Fotografía aérea del Muro Principal del Tranque Las Tórtolas, Marzo 2010. GEER Association Report №. GEER-022.

Figure 7. View of Las Tórtolas tailings dams from a helicopter, March 2010. GEER Association Report Nº. GEER-022.

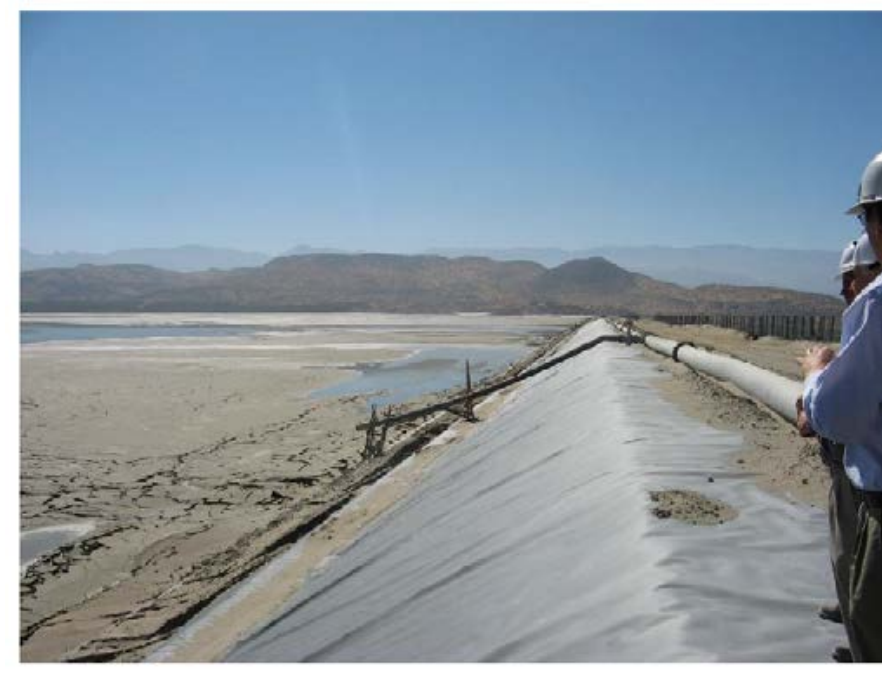

Figura 8. Vista del talud de aguas arriba del Muro del Tanque Ovejería. En la foto se observa que no existen daños en la geomembrana. GEER Association Report N . GEER-022.

Figure 8. View along the upstream face of the Ovejería tailings dam. There is no damage to the liner. GEER Association Report No. GEER-022.
Los muros de arenas de los tranques anteriormente descritos presentaron un comportamiento satisfactorio durante el terremoto de Maule. La geomembrana del talud de aguas arriba no presentó daños y no se observaron grietas ni en coronamiento ni en el talud de aguas abajo de los muros. En las Figuras 7 y 8 se muestran fotografías de los tranque tomadas en marzo del 2010. (GEER Association Report N. GEER-022).

Como resultado de las experiencias exitosas sobre el buen comportamiento de los muros de arenas y en general de los muros de los tranques y depósitos de relaves sometidos a solicitaciones sísmicas extremas, Valenzuela (2016) destaca las siguientes buenas prácticas:

1. Control estricto de los contenidos de finos de las arenas para garantizar valores de permeabilidad elevados y gen consecuencia que existe un contraste significante de permeabilidades entre las arenas del muro y las lamas a contener por el mismo.

2. Compactación de las arenas, generalmente sobre el $95 \%$ de la densidad máxima seca del Proctor Standard.

3. Diseñar taludes aguas abajo con relaciones 3,5:1 a 4:1 (Horizontal: Vertical), para facilitar la compactación y reducir la concentración de tensiones en la fundación.

4. Los drenes basales deben diseñarse conservadoramente, cubriendo un alto porcentaje del área de fundación del muro y considerando la posible rotura de partículas debido a los altos niveles de tensione que implican alturas elevadas de los muros.

5. Tratar las fundaciones para evitar su potencial licuación ante un evento sísmico.

6. La laguna operacional de los tranques debe mantenerse con un volumen mínimo y alejada de los muros.

7. Cubrir el talud aguas arriba de los muros con geomembrana como una protección extra ante eventuales flujos de crecidas que pudiesen alcanzar el talud de aguas arriba de los muros.

8. Aplicar criterios conservadores para determinar la revancha de los muros, considerando deformaciones estáticas, crecidas máximas probables, deformaciones sísmicas para condición de operación y cierre. 
9. Considerar obras de evacuación de crecidas robustas para manejar las crecidas.

10. Realizar diseños seguros, que cumplan con los criterios de estabilidad ante condición estática y dinámica durante el inicio de la operación y los sucesivos crecimientos de los muros.

A estas buenas prácticas y de acuerdo a la experiencia de la autora, se considera adecuado incluir las siguientes buenas prácticas:

1. Diseñar una red de instrumentos geotécnicos que permita registrar en línea el comportamiento de las variables geotécnicas críticas como niveles freáticos, desplazamiento de taludes, comportamiento de los sistemas de drenajes.

2. Durante la construcción realizar controles sobre la calidad geotécnica de los suelos que se utilizan para la construcción.

3. Controlar durante la construcción la humedad óptima y el grado de compactación de los suelos que conforman los muros.

4. Realizar topo-batimetría semestral para corroborar el cumplimiento de los planes de llenado previstos.

5. Generar Manuales de Operación y Planes de Emergencia definiendo umbrales aceptables e inaceptables de las variables críticas y acciones ante una eventual contingencia.

\section{Gestión de riesgos}

Aunque la normativa chilena, de manera implícita trata de minimizar y controlar los riesgos intrínsecos de los depósitos de relaves, actualmente se está incorporando un sistema de gestión de riesgo, siguiendo la gobernanza establecida por el ICMM (2016). Este sistema de gobernanza está basado en los siguientes seis elementos:

1. Establecer de manera clara las responsabilidades y competencias del dueño y realizar los proyectos con consultores que tengan el debido nivel de experiencia.

2. Planificación del depósito a largo plazo para asegurar los recursos incluyendo los humanos y los inversionales.

3. Gestionar los riesgos, lo que incluye identificar, mitigar y controlar tanto los riesgos técnicos como no técnicos.

4. Gestionar los cambios para prevenir anticipadamente los riesgos. Esto es, analizar y evaluar el impacto que tendrán los cambios en la evaluación de los riesgos.

5. Preparase para las emergencias, es decir contar con un manual de operaciones que indique qué acciones se deben tomar cuando el sistema de monitoreo registre variables fuera de rango. Esto último implica tener monitoreo en línea en tiempo real y definir los umbrales de control.

6. Revisar y garantizar el buen desempeño del depósito. Esto implica realizar revisiones frecuentes y auditorias. En este aspecto, actualmente, se han implementado la figura de Ingeniero de Registro; las Revisiones Expertas (Review Boards) y las Revisiones Independientes "Dam Safety Reviews" siguiendo las guías de la CAD, 2013.

El cumplir con los seis puntos mencionados anteriormente implica un gran desafío para la industria minera, y su implementación se está realizando de manera paulatina.

\section{El futuro de los relaves en Chile}

Actualmente, el Gobierno de Chile está desarrollando una Política Nacional Sobre Relaves, cuyos desafíos futuros son: generar nuevos paradigmas respecto a la gestión de relaves, dar una solución concreta a los pasivos ambientales mineros y crear una nueva industria a partir de residuos de la minería, introducción de la economía circular en el sector minero. Para concretar esta política el gobierno está financiando líneas de investigación como por ejemplo: un programa tecnológico de monitoreo en línea de depósitos de relaves cuyo objetivo principal es lograr el monitoreo en tiempo real y centralizado de los depósitos de relaves; líneas de I+D para recuperar elementos de valor de los relaves como por ejemplo cobalto, hierro, tierras raras; y programas de compensación de impactos a nuevos proyectos que se comprometan a trasladar 0 cerrar depósitos de relaves abandonados.

\section{Conclusiones}

Basado en la experiencia y estudio de las fallas de los depósitos de relave la normativa chilena ha evolucionado para asegurar el comportamiento y estabilidad de estas estructuras. Sin embargo, actualmente y debido a las últimas fallas catastróficas de Mont Polley, Samarco y Fundao se considera necesario establecer un sistema de gestión de riesgos basado en la gobernanza, gestión de cambios, gestión operacional preventiva-predictiva mejorando la comunicación y la respuesta entre las partes ante situaciones de eventuales emergencia y revisiones independientes externas que permitan mitigar y controlar los riesgos intrínsecos de los depósitos de relaves. El propósito final es diseñar y operar basados en el análisis de riesgos. Esto implica, determinar los criterios de diseños del depósito estableciendo primero las consecuencias de su falla.

También otros aspectos que importan son: disminuir el impacto de los relaves como pasivo ambiental a través de su reprocesamiento, lo permitirá disminuir su volumen y realizar el cierre seguro de los depósitos actualmente abandonados.

\section{Referencias}

Barrios, J.J. 2017. Análisis de Estabilidad de un depósito de relaves espesados emplazado sobre un tranque convencional. Tesis de pregrado. UniversidadTécnico Federico Santa María. 
Castro, G. and Troncoso, J. 1989. Effects of Chilean Earthquake on three tailings dams. International Seminar on Dynamic Behavior of Clays, Sands \& Gravel. Kitakyushu, Japan.

Dam Safety Guidelines, Canadian Dam Association, Toronto. 1999 (revised 2007 and 2013).

Decreto Supremo $N^{\circ}$ 50. 2015. Reglamento que establece las condiciones técnicas que deberán cumplirse en el proyecto, construcción y operación de las obras hidráulicas identificadas en el artículo 294 del código de aguas.

Decreto Supremo $N^{\circ}$ 86. 1970. Reglamento de construcción y operación de tranques de relaves. Ministerio de Minería de Chile.

Decreto Supremo $\mathrm{N}^{\circ}$ 248. Reglamento para la aprobación de proyectos de diseño, construcción, operación y cierre de depósitos de relaves. Ministerio de Minería de Chile.

Dobry, R. and Alvarez, L. 1967. Seismic Failures of Chilean Tailings Dams. Journal of Soil Mechanics and Foundation Division, ASCE ( 9):237-260.

GEER Association Report N GEER-022. 2010. Geo-Engineering Reconnaissance of the February 27, 2010 Maule, Chile Earthquake.

ICMM. 2016. Position Statement on Preventig Catastrophic Failure of Tailings Storage Facilities, International Council on Mining \& Metals, London.

Ley $N^{\circ}$ 20.551. 2011. Regula el Cierre de Faenas e Ins- talaciones Mineras.

Ley 19.300. 1994. Bases Generales del Medio Ambiente.

Sernageomin. 2018. Análisis de los Depósitos de Relaves en Chile, 05/2018, https://www.sernageomin.cl

Troncoso J.H., Avendaño A. y Vergara A. 1993. The Seismic Failure of Barahona Tailings Dam. Proceedings Third International Conference on Case Histories in Geotechnical Engineering, St. Louis, Missouri, June 1-4, paper $\mathrm{N}^{\circ} 256$.

Valenzuela, L. 1996. Main characteristics of large tailings dams. In Large Dams in Chile, ICOLD Chile annual meeting of ICOLD International, Santiago.

Valenzuela, L. 2016. Design, construction, operation and effect of fines content and permeability on the seismic performance of tailings sand dams in Chile. Obras y Proyectos 19:6-22.

Verdugo R., Sitarr N., Frost J., Bray J., Candia G., Eldrige T., Hashash Y., Olson S., Urzua A. 2012. Seismic Performance of Earth Structures during February 2010 Maule, Chile, Earthqueake: Dams, levees, Tailings Dams and Retaining Walls. Earthquake Spectra, 2018)(S1): S75-S96.

Villavicencio G., Espinace R., Palma J., Fourie A., Valenzuela P. 2014 Failures of sand tailings dams in a highly seismic country. Canadian Geotechnical Journal 51:449-464.

Recibido: julio 2020

Revisado: diciembre 2020

Aceptado: enero 2021

Publicado: diciembre 2021 
